\title{
Announcement
}

\section{Special Issue Planned}

Law and Human Behavior is planning a special issue dealing with discrimination and the law. Scientific research on issues of discrimination is increasingly introduced into legal settings. This work comes from a variety of disciplines and raises numerous issues. The planned issue will include articles based on original research as well as review essays, dealing with issues of sex, age, race, class, and other kinds of discrimination handled in various legal settings. The focus will be multi-disciplinary and, conceivably, cross-national.

Please send concept papers and/or manuscripts to the guest editor for this issue, John Hagan, Faculty of Law, University of Toronto, Toronto, Ontario, Canada M5S 1A1. 